Pacific Journal of Mathematic 


\title{
SOME ITERATIVE METHODS FOR DETERMINING ZEROS OF FUNCTIONS OF A COMPLEX VARIABLE
}

\author{
W. D. MunRo
}

1. Introduction. Innumerable schemes have been developed for finding approximate numerical values for the zeros of a function $f(z)$. It is remarkable, considering the diversity of motivating ideas behind these methods, that so many of them (including the classical Newton-Raphson method) are essentially nothing more than combinations of Bernoulli's method and, in effect, the origin shifting procedure of Horner. In turn these combinations may be formulated in terms of the power series expansion of a fraction $g(z) / f(z)=\sum_{n=0}^{\infty} c_{n} z^{n}$. We present here several methods of utilizing the information implicit in the coefficients $c_{n}$, obtaining several new iterative schemes for approximating zeros and reformulating some well known ones-frequently in such a way as to provide a simpler method of computation.

We assume that for computational purposes, if $g(z)=\sum_{n=0}^{\infty} a_{n} z^{n}$ and $f(z)=\sum_{n=0}^{\infty} b_{n} z^{n}$, then the coefficients for $g(z) / f(z)=\sum_{n=0}^{\infty} c_{n} z^{n}$ may conveniently be obtained recursively by the standard equations

$$
\begin{gathered}
c_{0} b_{0}=a_{0} \\
c_{0} b_{1}+c_{1} b_{0}=a_{1} \\
\cdots \cdots+\cdots \\
c_{0} b_{n}+c_{1} b_{n-1}+\cdots+c_{n} b_{0}=a_{n}
\end{gathered}
$$

where for convenience, we make $b_{0}=1$ so that each coefficient is given as a product sum $c_{n}=a_{n-1}-b_{1} c_{n-1}-\cdots-b_{n} c_{0}$.

By the order of an iteration we mean the concept introduced by Schröder [19]. If an iteration produces a sequence $z_{n} \rightarrow \alpha$, it is of order $N$ if

$$
\frac{\left|z_{n+1}-\alpha\right|}{\left|z_{n}-\alpha\right|^{N}} \rightarrow c \neq 0
$$

$c$ a constant.

2. Basic lemma. We base our results on a lemma which specifies the contribution to the coefficients $c_{n}$ of those zeros closest to the origin. Let $f(z)$ and $g(z)$ be analytic in $|z|<R$ and let $f(z)$ have zeros $\alpha_{\imath}, i=$

Received June 16, 1958. The preparation of this paper was sponsored by the Office of Naval Research. Reproduction in whole or in part is permitted for any purpose of the United States Government. 
$1, \cdots, K$, of order $m_{i}>0$ and such that $0<\left|\alpha_{i}\right|=a<R, f(z) \neq 0$ for $|z|<a$. Let $g(z)$ have zeros of order $n_{i} \geqq 0$ at $\alpha_{i}$ with $p_{i}=m_{i} n_{i}>0$. If $f(z)$ vanishes elsewhere in $|z|<R$, let $\beta_{i}, i=1, \cdots, L$ be those zeros of order $M_{i}>0$ for which $a<\left|\beta_{i}\right|=b<R, f(z) \neq 0$ in $a<|z|<b$ and for which $g(z)$ has zeros of order $N_{i} \geqq 0$ with $q_{i}=M_{i}-N_{i}>0$. If no such zeros exist in $|z|<R$ let $b$ be any value for which $a<b<R$. In either case let $\delta=a / b<1$.

Under the conditions specified

$$
\begin{aligned}
\frac{g(z)}{f(z)}= & \sum_{i=1}^{K}\left[\frac{A_{i 1}}{\left(z-\alpha_{i}\right)}+\cdots+\frac{A_{i p_{i}}}{\left(z-\alpha_{i}\right)^{p_{i}}}\right] \\
& +\sum_{i=1}^{L}\left[\frac{B_{i 1}}{\left(z-\beta_{i}\right)}+\cdots+\frac{B_{i q_{i}}}{\left(z-\beta_{i}\right)^{q_{i}}}\right]+\varphi(z) \\
= & \sum_{i=1}^{K}\left[\frac{A_{i 1}}{\left(z-\alpha_{i}\right)}+\cdots+\frac{A_{i p_{i}}}{\left(z-\alpha_{i}\right)^{p_{i}}}\right]+\psi(z) .
\end{aligned}
$$

In the event that no $\beta_{i}$ occurs, $B_{i j}=0$ and $\varphi(z)$ and $\psi(z)$ are identical. $\varphi(z)$ is analytic in $|z| \leqq b$ and $\varphi(z)=\sum_{n=0}^{\infty} A_{n} z^{n}$ has radius of convergence $R^{\prime}>b . \quad \psi(z)$ is analytic in $|z|<b$ and $\psi(z)=\sum_{n=0}^{\infty} B_{n} z^{n}$ has radius of convergence $R^{\prime \prime} \geqq b$. Direct expansion of the right hand side of (2) gives

$$
\begin{aligned}
\frac{g(z)}{f(z)} & =\sum_{n=0}^{\infty} c_{n} z^{n}, \\
c_{n} & =\sum_{i=1}^{K} \frac{P_{i}(n)}{\alpha_{i}^{n+1}}+\sum_{i=1}^{L} \frac{Q_{i}(n)}{\beta_{i}^{n+1}}+\mathrm{A}_{n} \\
& =\sum_{i=1}^{K} \frac{P_{i}(n)}{\alpha_{i}^{n+1}}+B_{n} .
\end{aligned}
$$

The functions $P_{i}(n)$ and $Q_{i}(n)$ are polynomials

$$
\begin{aligned}
& P_{i}(n)=\sum_{k=1}^{p_{i}} \frac{(-1)^{k} A_{i k}(n+1)^{|k|}}{\alpha_{i}^{k}(k-1) !}, \\
& Q_{i}(n)=\sum_{k=1}^{q_{i}} \frac{(-1)^{k} B_{i k}(n+1)^{|k|}}{\beta_{i}^{k}(k-1) !}
\end{aligned}
$$

with $(n+1)^{|k|}=(n+1)(n+2) \cdots(n+k)$ and $(n+1)^{|0|}=1$.

Lemma 1. Under the conditions specified above the coefficients $A_{n}$ and $B_{n}$ of (3) satisfy

$$
a^{n+1}\left|A_{n}\right|<C \delta^{n+1}
$$

and 


$$
a^{n+1}\left|B_{n}\right|<D \Delta^{n+1}
$$

for $\delta<\Delta<1$,

$C$ and $D$ being constants independent of $n$.

Proof. The proof is immediate by Cauchy's inequality and the remarks concerning the radii of convergence $R^{\prime}$ and $R^{\prime \prime}$. Since $R^{\prime}>b$, $\left|A_{n}\right|<C / b^{n+1}$ and $a^{n+1}\left|A_{n}\right|<C \delta^{n+1}$. For any $\delta<\Delta<1, \Delta=a / b^{\prime}, a<b^{\prime}<b$ and $R^{\prime \prime} \geqq b>b^{\prime}$ and as before Cauchy's inequality gives (5).

If each $q_{i}=1$, then $Q_{i}(n)$ is constant, and $B_{n}$ satisfies an inequality like (4). If $f(z) / g(z)$ is a rational fraction with only simple poles, we may in (5) take $\Delta=\delta$, and $D$ as the number of poles in $|z|>a$.

It is apparent in (3) that the use of $c_{n}$ to give information concerning the $\alpha_{i}$ is dependent on the smallness of $B_{n}$ which is governed by inequality (5). $B_{n}$ may be made smaller in a variety of ways. The most obvious method is to increase $n$. Alternately we may attempt to decrease $\Delta$ or $D$ by means of a transformation or by alternation of $g(z)$. We consider these methods in turn.

3. Methods of Bernoulli and Whittaker. Of particular interest is the case $K=1, \alpha_{i}=\alpha, p_{i}=p, P_{i}(n)=P(n)$, for which

$$
c_{n}=\frac{P(n)}{\alpha^{n+1}}+B_{n}=\frac{1}{\alpha^{n+1}}\left[P(n)+O\left(\Delta^{n+1}\right)\right]
$$

as $n \rightarrow \infty$, by Lemma 1 . Hence

$$
\frac{c_{n}}{c_{n+1}}=\alpha \frac{P(n)+O\left(\Delta^{n+1}\right)}{P(n+1)+O\left(\Delta^{n+1}\right)} \rightarrow \alpha,
$$

which is König's theorem [12].

In the case of a polynomial, use of $c_{n} / c_{n+1}$ to approximate $\alpha$ is equivalent to Bernoulli's method (applied to $f(1 / z)$ ) with a more or less arbitrary choice of $g(z)$. With $g(z)=1, c_{n} / c_{n+1}$ is the basis for Whittaker's formula [20].

It is worth noting that the rate of convergence of the BernoulliWhittaker method is slower if either $p>1$ or some $q_{i}>1$, that is if $\alpha$ or any of the zeros next closer to the origin yield other than a simple pole. If $p=q_{i}=1$, the error is $O\left(\delta^{n+1}\right)$ as $n \rightarrow \infty$, since $P(n)$ and $Q_{i}(n)$ are constant. On the other hand if $p>1$ the error is $O(1 / n)$. Even with $p=1$ if any $q_{i}>1$, the presence of the polynomial $Q_{i}(n)$ in $B_{n}$ slows convergence, the error being $O\left(\Delta^{n+1}\right), \Delta>\delta$. For this reason, the choice $g(z)=f^{\prime}(z)$ is best since it guarantees $P(n)=Q_{i}(n)=1$.

The possibility of using the $c_{n}$ to find all $\alpha_{i}, K>1$, and indeed to find all zeros of $f(z)$ in a region was suggested by Hadamard [4], and such a generalization of the basic Bernoulli method has been given by 
Aitken [1, 2], Golomb [3], Henrici [6] and Rutishauser [18]. We concern ourselves here with refinements applicable to finding a single zero of smallest modulus or a conjugate complex pair.

4. Generalizations of Newton's method. Again let $K=1$. Then any transformation which will decrease $\delta$ and $\Delta$ in (4) and (5) without radically increasing $C$ or $D$ will improve the accuracy of $c_{n} / c_{n+1}$ as an approximation to $\alpha$ for given $n$. The most obvious transformation is a shift of the origin to decrease $a=|\alpha|$. This scheme was considered for a simple zero by Housholder [10] and in the general case by Kulik [13]. We present here a theorem giving the order of convergence explicitly in terms of the value of $p$.

THEOREM 1. Let $f(z)$ and $g(z)$ be analytic in some neighborhood of $z=\alpha$, a zero of order $m>0$ for $f(z)$ and $n \geqq 0$ for $g(z)$ with $p=m-n>0$. Let

$$
\frac{g(w+z)}{f(w+z)}=\sum_{n=0}^{\infty} c_{n}(z) w^{n}
$$

be the Taylor expansion about a point $z$ in the neighborhood of $\alpha$, and for fixed $N$, define the iteration

$$
z_{n+1}=z_{n}+\frac{c_{N}\left(z_{n}\right)}{c_{N+1}\left(z_{n}\right)}=\varphi\left(z_{n}\right) .
$$

Then there is always a $\rho>0$ such that if $\left|z_{0}-\alpha\right|<\rho, z_{n} \rightarrow \alpha$ as $n \rightarrow \infty$. If $p=1$ the order of the iteration is at least $N+2$. If $p>1$ the order of the iteration is one.

Proof. For $p=1$ we have as in (3),

$$
c_{k}(z)=\frac{A}{(\alpha-z)^{k+1}}+B_{k}(z),
$$

so that

$$
\begin{aligned}
\varphi(z) & =z+\frac{c_{N}(z)}{c_{N+1}(z)}=z-\frac{A(z-\alpha)-B_{N}(z)(\alpha-z)^{N+2}}{A+B_{N+1}(z)(\alpha-z)^{N+2}} \\
& =z-\frac{P(z)}{Q(z)} .
\end{aligned}
$$

Obviously $\alpha=\phi(\alpha)$, since $P(\alpha)=0, Q(\alpha) \neq 0$. We also have $P^{\prime}(\alpha)=A=$ $Q(\alpha) \neq 0$ and

$$
P^{(k)}(\alpha)=0=k Q^{(k-1)}(\alpha), \quad k=2, \cdots, N+1
$$


and by a theorem of Ludwig [16] there is a $\rho>0$ such that $\left|z_{0}-\alpha\right|<\rho$ implies convergence of $\left\{z_{n}\right\}$ to $\alpha$ and the order of the iteration is at least $N+2$. Since

$$
p^{(N+2)}(\alpha)=(N+2) B_{N}(\alpha), Q^{(N+1)}(\alpha)=0 .
$$

The order is exactly $N+2$ unless $B_{N}(\alpha)=0$.

For $p>1$ we have

$$
c_{k}(z)=\frac{P_{k}(z)}{(\alpha-z)^{k+p}}+B_{k}(z)
$$

with

$$
P_{k}(z)=\sum_{i=0}^{p-1} \frac{(-1)^{i} A_{i+1}(\alpha-z)^{p-i-1}(k+1)^{|i|}}{k !} .
$$

Hence

$$
\varphi(z)=z-\frac{(z-\alpha) P_{N}(z)+B_{N}(z)(\alpha-z)^{N+p+1}}{P_{N+1}(z)+B_{N+1}(z)(\alpha-z)^{N+p+1}},
$$

and

$$
\phi^{\prime}(\alpha)=1-\frac{P_{N}(\alpha)}{P_{N+1}(\alpha)}=1-\frac{N+1}{N+p}
$$

so that $0<\varphi^{\prime}(\alpha)<1$ and convergence occurs in some neighborhood of $\alpha$, the order of the iteration being one.

Since the introduction of $f^{\prime}(z)$ into $g(z)$ guarantees no multiple poles, we have the following.

CoROLlaRY. If $g(z)=h(z) f^{\prime}(z), h(\alpha) \neq 0$, then $p=1$ and the order of the iteration is at least $N+2$.

The iteration may be written explicitly in terms of $f(z)$ and $g(z)$ as

$$
\varphi(z)=z-f(z) \frac{D_{N}(z)}{D_{N+1}(z)},
$$

with

$$
D_{N}(z)=\left|\begin{array}{llllll}
f(z) & 0 & 0 & \cdots & 0 & g(z) \\
f^{\prime}(z) & f(z) & 0 & \cdots & g^{\prime}(z) \\
\frac{f^{\prime \prime}(z)}{2 !} & f^{\prime}(z) & f(z) & \cdots & \frac{g^{\prime \prime}(z)}{2 !} \\
& \cdots \cdots \cdots \cdots & \\
\frac{f^{(N)}(z)}{N !} \frac{f^{(N-1)}(z)}{(N-1) !} \frac{f^{(N-2)}(z)}{(N-2) !} \cdots f^{\prime}(z) & \frac{g^{((N)}(z)}{N !}
\end{array}\right| .
$$


For $g(z)=1, N=0$ we have Newton's method. For $N=1,2,3,4$ we have iterations given by Hitcheock $[7,8,9]$, Kiss [11] and Richmond [17]. For the general case of $g(z)=1$ the iterations given by Hamilton [5] and Zajta [21] are equivalent to these but give computation only in terms of the evaluation of the determinants (7). From this point of view the higher order iterations are of more theoretical than practical value. In the form (6), however, it is apparent that all that is required for one cycle is a shift of the origin (perhaps by Horner's method) and computation of the necessary $c_{k}(z)$ recursively by (1), so that computation is simple and easily coded for high speed machines. In particular if $g(z)=1$ or $g(z)=f^{\prime}(z)$ (which is better, certainly, for multiple zeros) only one origin shift need be made since the coefficients in the numerator are automatically given.

An alternative transformation may be used to achieve the same order of iteration with somewhat simpler computation.

THEOREM 2. Let $f(z)$ and $g(z)$ be analytic in some neighborhood of $z=\alpha, a$ zero of order $m>0$ for $f(z)$ and $n \geqq 0$ for $g(z)$ with $p=m-n>0$. Let

$$
\frac{g[z(w+1)]}{f[z(w+1)]}=\sum_{n=0}^{\infty} c_{n}(z) w^{n}
$$

be the Taylor expansion about $w=0$ and for fixed $N$ define the iteration

$$
z_{n+1}=z_{n}\left(1+\frac{c_{N}(z)}{c_{N+1}(z)}\right)=\varphi\left(z_{n}\right) .
$$

Then there is always $a \rho>0$ such that if $\left|z_{0}-\alpha\right|<\rho, z_{n} \rightarrow \alpha$ as $n \rightarrow \infty$. If $p=1$ the order of the iteration is at least $N+2$. If $p=1$ the order of the iteration is at least $N+2$. If $p>1$ the order of the iteration is one.

The proof parallels exactly the proof for Theorem 1. The iterations of Theorem 2 have some advantage in computation, since the coefficients in $g[z(w+1)]$ and $f[z(w+1)]$ can be generated by successive transformations, forming $g(z u)$ and $f(z u)$ and shifting the origin by $u=w+1$. This has the advantage that no multiplications are involved in an origin shift to the point 1 by Horner's method. For a second order iteration (as Newton's method for example) the number of multiplications for the two methods is essentially the same. For higher order iterations, however, no additional multiplications are required in the method of Theorem 2 to generate all the necessary coefficients of $g[z(w+1)]$ and $f[z(w+1)]$. Again if $g(z)=h(z) f^{\prime}(z), h(\alpha) \neq 0$, no multiple pole occurs and the order of the iteration is at least $N+2$. 
5. Variation of the numerator. In the preceding section, use was made of transformations to improve the value of $\delta$. An alternative scheme is to decrease the contribution of $B_{n}$ in (3) by variation of $g(z)$. Ideally, if $f(z)=(z-\alpha)^{m} Q(z), Q(\alpha) \neq 0$, a choice of $g(z)=Q(z)$ would make $B_{n}=0$. Since $Q(z)$ is not known we attempt to approximate it. Several rather simple iterations result, which though theoretically applicable to any analytic function, are more practical in the case of polynomials because of the simplicity of performing the required division.

Lemma 2. Let $f(z)$ be analytic in $|z|<R$ and let $\alpha \neq 0$ be a simple zero of $f(z)$ such that $f(z) \neq 0$ in $|z| \leqq|\alpha|$ except at $\alpha$ itself. Let $\Delta<1$ be defined as in Lemma 1 . Let $Q_{z}(w)=(f(w)-f(z)) /(w-z)$, and

$$
\frac{Q_{z}(w)}{f(w)}=\sum_{n=0}^{\infty} c_{n}(z) w^{n}
$$

be the Taylor expansion about $W=0$. The functions

$$
\begin{array}{ll}
\psi(z)=-\frac{1}{c_{0}(z)} & \\
\varphi_{N}(z)=\frac{c_{N}(z)}{c_{N+1}(z)} & \text { for fixed } N \geqq 0
\end{array}
$$

satisfy the conditions $\psi(\alpha)=\alpha, \varphi_{N}(\alpha)=\alpha$ and $\left|\varphi_{N}^{\prime}(\alpha)\right|<P \Delta^{N+2}, P$ a constant.

Proof. The condition $\psi(\alpha)=\alpha$ is obvious since $c_{0}(z)=(f(0)-f(z)) /-z f(0)$, so that $c_{0}(\alpha)=-1 / \alpha$. More generally,

$$
\frac{Q_{z}(w)}{f(w)}=\frac{1}{w-z}\left[1-\frac{f(z)}{f(w)}\right]
$$

and if

$$
[f(z)]^{-1}=\sum_{k=0}^{\infty} A_{k} z^{k}
$$

with

$$
S_{n}(z)=\sum_{k=0}^{n} A_{k} z^{k}
$$

then

$$
c_{n}(z)=\left(f(z) S_{n+1}(z)-1\right) / z^{n+1}
$$

Hence

$$
\varphi_{N}(z)=z \frac{1-f(z) S_{N}(z)}{1-f(z) S_{N+1}(z)}
$$


so that $\rho_{N}(\alpha)=\alpha$. Moreover

$$
\varphi_{N}^{\prime}(\alpha)=1+f^{\prime}(\alpha) A_{N+1} \alpha^{N+2}
$$

with

$$
A_{N+1}=-\left[f^{\prime}(\alpha) \alpha^{N+2}\right]^{-1}+B_{N+1},
$$

and $\varphi_{N}^{\prime}(\alpha)=f^{\prime}(\alpha) \alpha^{N+2} B_{N+1}$. By Lemma 1, however, $\left|\alpha^{N+2} B_{N+1}\right|<D \Delta^{N+2}$, and

$$
\left|\varphi_{N}^{\prime}(\alpha)\right|<D\left|f^{\prime}(\alpha)\right| \Delta^{N+2}=P \Delta^{N+2} .
$$

The function $\psi(z)$ defines an iteration $z_{n+1}=\psi\left(z_{n}\right)$ which is equivalent to one of $\operatorname{Lin}[14,15]$. The function $\varphi_{N}(z)$ defines iterations $z_{n+1}=$ $\mathcal{P}_{N}\left(z_{n}\right)$. Convergence in the latter case can always be achieved in either one of two ways. By Lemma 2, since $\Delta<1,\left|\varphi_{N}^{\prime}(\alpha)\right|<1$ if $N$ is large enough. Alternately an initial shift of the origin to a point close enough to $\alpha$ provides a value of $\Delta$ sufficiently small to make $\left|\varphi_{N}^{\prime}(\alpha)\right|<1$ for any choice of $N$. In any case the computation of a single cycle consists of a single synthetic division by $w-z_{n}$ and computation of $c_{k}\left(z_{n}\right)$ by (1).

For large $N$ the computation may be greater than the rapidity of convergence would warrant. An alternative and simpler iteration may be obtained. The function $\varphi_{N}(z)$ may be written as

$$
\varphi_{N}(z)=z\left(1+\frac{A_{N+1} z^{N+1} f(z)}{1-f(z) S_{N+1}(z)}\right) .
$$

If now the denominator is taken as 1 , there results

$$
\psi_{N}(z)=z+A_{N+1} z^{N+2} f(z)
$$

with the property that $\psi_{r_{N}}(\alpha)=\alpha,\left|\psi_{N}^{\prime}(\alpha)\right|<P \Delta^{N+2}$, by the same argument as before. For $N$ large enough or $\Delta$ small enough $\left|\psi_{N}^{\prime}(\alpha)\right|<1$ and there is a neighborhood of $\alpha$ in which the iteration $z_{n+1}=\psi_{N}\left(z_{n}\right)$ converges to $\alpha$. Since $\psi_{N}(z)$ is a polynomial this yields a particularly simple iteration involving no division.

Although the functions $\psi(z)$ and $\psi_{N}(z)$ satisfy $\alpha=\psi(\alpha)$ and $\alpha=\psi_{N}(\alpha)$ for a zero of order higher than one, the inclusion of the polynomial term in $A_{N+1}$ may prevent convergence.

For zeros occurring in conjugate complex pairs a similar iteration may be used with synthetic division by a quadratic factor. If $w^{2}+p_{n} w+q_{n}$ is an approximate quadratic factor for the roots $\alpha \pm i \beta$ we define $Q_{n}(w)$ by

$$
f(w)=\left(w^{2}+p_{n} w+q_{n}\right) Q_{n}(w)+A_{n} w+B_{n} .
$$

The expansion 


$$
\frac{Q_{n}(w)}{f(w)}=\sum_{k=0}^{\infty} c_{k}\left(p_{n}, q_{n}\right) w^{k}
$$

approximates that of $\left[w^{2}+p w+q\right]^{-1}$ for the true quadratic factor. The new values of $p_{n+1}$ and $q_{n+1}$ are obtainable by solving a pair of simultaneous linear equations, the simplest of which gives $q_{n+1}=\left[c_{0}\left(p_{n}, q_{n}\right)\right]^{-1}$ and $p_{n+1}=-c_{1}\left(p_{n}, q_{n}\right)\left[c_{0}\left(p_{n}, q_{n}\right)\right]^{-2}$.

6. Combined methods. The methods considered in the preceding sections may be combined to give still higher order iterations with essentially no increase in computation. In particular we may combine the transformations of Theorems 1 and 2 with the quotient method of Lemma 2. This gives the following iterations.

THEOREM 3. Let $f(z)$ and $g(z)$ be analytic in some neighborhood of $z=\alpha$, a zero of order $m>0$ for $f(z)$ and $n \geqq 0$ for $g(z)$ with $p=m-n>0$. Let

$$
Q_{z}(w)=\frac{f(w)-f(z)}{w-z}, F_{z}(w)=f(z w), R_{z}(w)=\frac{F_{z}(w)-F_{z}(1)}{w-1}
$$

and let

$$
\frac{Q_{z}(w+z)}{f(w+z)}=\sum_{n=0}^{\infty} c_{n}(z) w^{n}
$$

and

$$
\frac{R_{z}(w+1)}{F_{z}(w+1)}=\sum_{n=0}^{\infty} C_{n}(z) w^{n}
$$

be the appropriate Taylor expansions, and for fixed $N$ define the iterations

$$
z_{n+1}=z_{n}+\frac{c_{N}\left(z_{n}\right)}{c_{N+1}\left(z_{n}\right)}=\mathcal{p}\left(z_{n}\right)
$$

and

$$
z_{n+1}=z_{n}\left[1+\frac{C_{N}\left(z_{n}\right)}{C_{N+1}\left(z_{n}\right)}\right]=\psi_{r}\left(z_{n}\right)
$$

Then for each iteration there is a $\rho>0$ such that if $\left|z_{0}-\alpha\right|<\rho z_{n} \rightarrow \alpha$ as $n \rightarrow \infty$. If $p=1$ the order of the iteration is at least $N+3$. If $p>1$ the order of the iteration is one.

Proof. We have in the first case 


$$
\begin{aligned}
\frac{Q_{z}(w+z)}{f(w+z)} & =\frac{1}{w}\left[1-\frac{f(z)}{f(w+z)}\right] \\
& =\sum_{n=0}^{\infty} f(z) b_{n+1}(z) w^{n}
\end{aligned}
$$

where $[f(w+z)]^{-1}=\sum_{n=0}^{\infty} b_{n}(z) w^{n}$. Hence

$$
\varphi(z)=z+\frac{b_{N+1}(z)}{b_{N+2}(z)}
$$

which by Theorem 1 establishes the result. A similar result holds for $\psi(z)$ with Theorem 2.

It is important to note here that all the coefficients of $Q_{z}(w+z)$ are automatically generated in the transformation $f(w+z)$, and hence there is a gain of one in the order of the iteration by using already computed values in the recursion relations (1). Similarly after the preliminary transformation $F_{z}(w)=f(z w)$ the coefficients for both $R_{z}(w+1)$ and $F_{z}(w+1)$ are computed simultaneously with no additional multiplications and there is again a gain of one in the order of the iteration.

7. Computational aspects. From the point of view of explicit formulation in terms of $f(z)$ and $g(z)$ the higher order iterations given in Theorems 1, 2 and 3 appear so hopelessly complicated as to be of no practical value. If it is remembered, however, that each of these iterations, including the classical Newton one, consists in each cycle of a transformation and computation of product sums by (1), the apparent simplicity of the lower order iterations is not so evident. In the end it is a matter basically of applying the Bernoulli method (with appropriate initial conditions), using a periodic transformation to speed convergence. From this point of view the choice of a given iteration will depend on the relative simplicity of the operations in (1) and in the transformation. For machines with a single command to form product sums of $m$ pairs of numbers, there are advantages in using higher order iterations and cutting down the number of transformations.

There are other reasons why the Newton iteration, in spite of its apparent simplicity, may not be the best choice. It may well be that a somewhat unrefined first approximation is not good enough for convergence with a lower order iteration but is for a higher order one. This is not surprising if we recall that the first step in an iteration of any order is just the Bernoulli method carried that far, yielding, itself, a first approximation-and in this case Newton's iteration may not be quite good enough. A simple example is given by the function $f(z)=2 z^{3}-9 z^{2}+11 z-3$. Since $f(1)>0, f(2)<0$, a reasonable guess might be $z_{0}=1$ or $z_{0}=2$ as a starting value. Newton's method (corresponding to $g(z)=1$ ) gives here 
the useless oscillating sequence $1,2,1,2, \cdots$. On the other hand the third order iteration with $z_{0}=1$ (and little additional work) starts the sequence $1,1.25,1.45, \cdots$ which is converging to the true root, $3 / 2$.

It is interesting to note here that the second order iteration corresponding to $g(z)=f^{\prime}(z)$ and $z_{0}=1$ does converge, starting $1,1.14, \cdots$. The reason for this is apparent if one considers that in the early stages, even for simple zeros, it is likely to be the coefficients of the form $A_{i 1}$ and $B_{i 1}$ in (2) which dominate, rather than the value of $\delta$, and $g(z)=$ $f^{\prime}(z)$ guarantees values of unity for these.

In the case of multiple zeros, the choice $g(z)=f^{\prime}(z)$ rather than $g(z)=1$ is obviously best, even if one is using only the quadratically convergent methods, since it is only the first choice which preserves the quadratic convergence. Another situation which is similar to this is the case of a cluster of zeros close together which will appear at relatively low accuracy as a multiple zero. In such a case, while the quadratic convergence of Newton's method is retained, convergence may be slower. A simple example is given by $f(z)=1-1.99 x+.99 x^{2}$, which has zeros 1 and $100 / 99=1.0101 \cdots$ close together. Using $z_{0}=0$ we have by Newton's method $(g(z)=1), z_{1}=.502$ to three places, while $g(z)=f^{\prime}(z)$ gives $z_{1}=1.005$. Even with the much better $z_{0}=.9, g(z)=1$ gives $z_{1}=$ .952 while $g(z)=f^{\prime}(z)$ yields $z_{1}=1.006$.

\section{REFERENCES}

1. A. C. Aitken, On Bernoulli's numerical solution of algebraic equations, Proc. Royal Soc. Edinburgh, 46 (1926), 289-305.

2. - Further numerical studies in algebraic equations, Proc. Royal Soc. Edinburgh, 51 (1931), 80-90.

3. M. Golomb, Zeros and poles of functions defined by Taylor series, Bull. Amer. Math. Soc., 46 (1943), 450-452.

4. J. Hadamard, Essai sur l'étude des fonctions donnécs par le développement de Taylor, J. Math., 4 (1892), 101-186.

5. H. J. Hamilton, Roots of equations by functional iteralion, Duke Math. J., 13 (1946), 113-121.

6. P. Henrici, The quotient-difference algorithm, National Bureau of Standards Appl. Math. Ser., 49 (1958), 23-46.

7. F. L. Hitchcock, Finding complex roots of equations, J. Math. Physics, 17 (1938), 5558.

8. - Algebraic equations with complex roots, J. Math. Phys., 18 (1939), 202-220.

9. - An improvement on the G. C. D. method for complex roots, J. Math, Phys., 23 (1944), 69-74.

10. A. Householder, Principles of numerical analysis, McGraw-Hill, New York, (1953), $123-125$.

11. I. Kiss, Über eine Varallgemeinerung des Newtonschen Näherungsfahren, Z. angew Math. Mech., 34 (1954), 68-69.

12. J. König, Ueber eine Eigenschaft der Potenzreihen, Math. Ann., 23 (1884), 447-449.

13. S. Kulik, A method for approximating the zeros of analytic functions, Duke Math. J., 24 (1957), 137-141. 
14. S. Lin. A Method of successive approximations for evaluating real and complex roots of cubic and higher order equations, J. Math. Phys., 20 (1941), 231-242.

15. - A method of finding roots of algebraic equations, J. Math, Phys., 22 (1943), 60-77.

16. R. Ludwig, Über Iterationsverfahren für Gleichungen und Gleichungs-system, Z. angew. Math. Mech., 34 (1954), 210-225.

17. H. W. Richmond, On certain formulas for numerical approximation, J. London Math. Soc., 19 (1944), 31-38.

18. H. Rutishauser, Eine Formel von Wronski und ihre Bedeutung für den QuotientenDifferenzen-Algoriihtmus, Z. angew. Math. Phys., 7 (1956), 164-165.

19. E. Schröder, Über unendliche viele Algorithmen zur Auflösung der Gleichungen, Math. Ann., 2 (1870), 317-365.

20. E. T. Whittaker, $A$ formula for the solution of algebraic and transcendental equations, Proc. Edinburgh Math. Soc., 36 (1918), 103-106.

21. A. Zajta, Unterschungen über die Verallgemeinerung der Newton-Raphson Wurzelapproximation, Acta Technica Acad. Sci. Hungaricae, 15 (1956), 233-260.

UNIVERSITY OF CALIFORNIA, LOS ANGELES

UNIVERSITY OF MINNESOTA 


\section{PACIFIC JOURNAL OF MATHEMATICS}

\section{EDITORS}

\section{David Gilbarg}

Stanford University

Stanford, California

\section{R. A. Beaumont}

University of Washington

Seattle 5 , Washington

\author{
A. L. Whiteman
}

University of Southern California Los Angeles 7, California

L. J. Paige

University of California

Los Angeles 24, California

\author{
E. F. BECKENBACH \\ C. E. BURGESS \\ E. HEWITT \\ A. HORN
}

\author{
V. GANAPATHY IYER \\ R. D. JAMES \\ M. S. KNEBELMAN \\ L. NACHBIN
}

ASSOCIATE EDITORS
I. NIVEN

T. G. OSTROM

H. L. ROYDEN

M. M. SCHIFFER
E. G. STRAUS

G. SZEKERES

F. WOLF

K. YOSIDA

\section{SUPPORTING INSTITUTIONS}

\author{
UNIVERSITY OF BRITISH COLUMBIA \\ CALIFORNIA INSTITUTE OF TECHNOLOGY \\ UNIVERSITY OF CALIFORNIA \\ MONTANA STATE UNIVERSITY \\ UNIVERSITY OF NEVADA \\ OREGON STATE COLLEGE \\ UNIVERSITY OF OREGON \\ OSAKA UNIVERSITY \\ UNIVERSITY OF SOUTHERN CALIFORNIA
}

\author{
STANFORD UNIVERSITY \\ UNIVERSITY OF TOKYO \\ UNIVERSITY OF UTAH \\ WASHINGTON STATE COLLEGE \\ UNIVERSITY OF WASHINGTON \\ * * * \\ AMERICAN MATHEMATICAL SOCIETY \\ CALIFORNIA RESEARCH CORPORATION \\ HUGHES AIRCRAFT COMPANY \\ SPACE TECHNOLOGY LABORATORIES
}

Mathematical papers intended for publication in the Pacific Journal of Mathematics should be typewritten (double spaced), and the author should keep a complete copy. Manuscripts may be sent to any one of the four editors. All other communications to the editors should be addressed to the managing editor, L. J. Paige at the University of California, Los Angeles 24, California.

50 reprints per author of each article are furnished free of charge; additional copies may be obtained at cost in multiples of 50 .

The Pacific Journal of Mathematics is published quarterly, in March, June, September, and December. The price per volume (4 numbers) is $\$ 12.00$; single issues, $\$ 3.50$. Back numbers are available. Special price to individual faculty members of supporting institutions and to individual members of the American Mathematical Society: $\$ 4.00$ per volume; single issues, $\$ 1.25$.

Subscriptions, orders for back numbers, and changes of address should be sent to Pacific Journal of Mathematics, 2120 Oxford Street, Berkeley 4, California.

Printed at Kokusai Bunken Insatsusha (International Academic Printing Co., Ltd.), No. 6, 2-chome, Fujimi-cho, Chiyoda-ku, Tokyo, Japan.

PUBLISHED BY PACIFIC JOURNAL OF MATHEMATICS, A NON-PROFIT CORPORATION

The Supporting Institutions listed above contribute to the cost of publication of this Journal, but they are not owners or publishers and have no responsibility for its content or policies. 


\section{Pacific Journal of Mathematics}

\section{Vol. 9, No. $2 \quad$ June, 1959}

Lee William Anderson, On the breadth and co-dimension of a topological lattice

Frank W. Anderson and Robert L. Blair, Characterizations of certain lattices

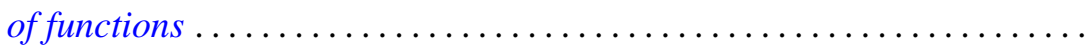

Donald Charles Benson, Extensions of a theorem of Loewner on integral

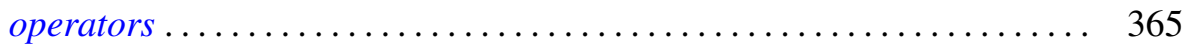

Errett Albert Bishop, A duality theorem for an arbitrary operator ........ 379

Robert McCallum Blumenthal and Ronald Kay Getoor, The asymptotic distribution of the eigenvalues for a class of Markov operators ........

Delmar L. Boyer and Elbert A. Walker, Almost locally pure Abelian

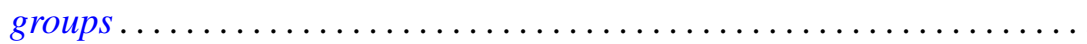

Paul Civin and Bertram Yood, Involutions on Banach algebras ........... Lincoln Kearney Durst, Exceptional real Lehmer sequences .... 415

Eldon Dyer and Allen Lowell Shields, Connectivity of topological

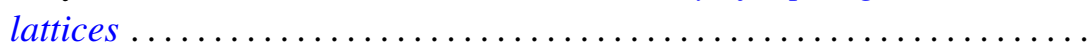

Ronald Kay Getoor, Markov operators and their associated

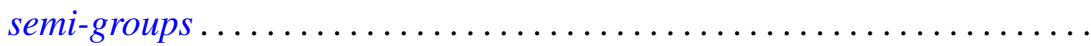

Bernard Greenspan, A bound for the orders of the components of a system of algebraic difference equations

Branko Grünbaum, On some covering and intersection properties in

Minkowski spaces ............................

Bruno Harris, Derivations of Jordan algebras ..............

Henry Berge Helson, Conjugate series in several variables.

Isidore Isaac Hirschman, Jr., A maximal problem in harmonic analysis.

II .

Alfred Horn and Robert Steinberg, Eigenvalues of the unitary part of a matrix

Edith Hirsch Luchins, On strictly semi-simple Banach algebras ...

William D. Munro, Some iterative methods for determining zeros of

functions of a complex variable...

John Rainwater, Spaces whose finest uniformity is metric .

William T. Reid, Variational aspects of generalized convex functions ....

A. Sade, Isomorphisme d'hypergroupoï des isotopes ...... . .

Isadore Manual Singer, The geometric interpretation of a special

connection . . .

Charles Andrew Swanson, Asymptotic perturbation series for characteristic

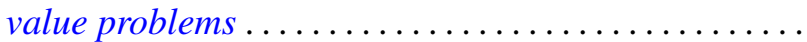

